\title{
Total variation as a metric for complementarity in energy resources time series
}

\author{
Diana Cantor ${ }^{1, *}$ Andrés Ochoa ${ }^{1}$ and Oscar Mesa ${ }^{1}$ \\ ${ }^{1}$ Universidad Nacional de Colombia, Medellín \\ ${ }^{*}$ Corresponding author: \\ Diana Cantor \\ dccantor@unal.edu.co
}

\begin{abstract}
Complementarity has become an essential concept in energy supply systems. Although there are some other metrics, most studies use correlation coefficients $(\rho)$ to quantify complementarity. The standard interpretation is that a high negative correlation indicates a high degree of complementarity. However, we show that the correlation is not an entirely satisfactory measure of complementarity. As an alternative, we propose a new index based on the mathematical concept of the total variation. For two time series, the new index $\phi$ is one minus the ratio of the total variation of the sum to the sum of the two series' total variation. We apply the index first to an auto-regressive $(\mathrm{AR})$ process and then to various Colombian electric system series. The AR case clearly illustrates the limitations of the correlation coefficient as a measure of complementarity. We then evaluate complementarity across various space-time scales in the Colombian power sectors, considering hydro and wind projects. The complementarity assessment on a broad temporal and geographical scale helps analyze large power systems with different energy sources. The case study of the Colombian hydropower systems suggests that $\phi$ is better than $\rho$ because (i) it considers scale, whereas $\rho$, being non-dimensional, is insensitive to the scale and even to the physical dimensions of the variables; (ii) one can apply $\phi$ to more than two resources; and (iii) $\rho$ tends to overestimate complementarity.
\end{abstract}

Keywords: electricity supply, natural resources, climatology, hydrology, electricity markets, reliability, flexibility

\section{Introduction}

The proliferation of energy sources caused by technological advances and their consequent cost reduction brought the concept of complementarity to the energy jargon. This concept is of particular interest when the resource's availability or their costs are variable in time. Although, up to now, there is no formal definition of resource complementarity in energy supply systems, intuition suggests that complementarity refers to the possibility of alternatively using different resources according to their availability.

Correlation coefficients are by far the most widely used metric for assessing complementarity between energy sources time series. This approach has been used, for example, in Poland (Jurasz, 2017), Portugal (Moura and de Almeida, 2010; Castro and Crispim, 2018), Spain (Ren et al., 2019),
Italy (Monforti et al., 2014; François et al., 2014), USA (Slusarewicz and Cohan, 2018), Colombia (Parra et al., 2020; Peña Gallardo et al., 2020b; Henao et al., 2020; Cantor et al., 2019), Germany (Schindler et al., 2020), Australia (Kay, 2015), Brazil (Silva et al., 2016; de Oliveira Costa Souza Rosa et al., 2017; dos Anjos et al., 2015; Cantão et al., 2017), China (Xu et al., 2017; Li et al., 2019; Cao et al., 2019), Sweden (Widen, 2011), Mexico (Peña Gallardo et al., 2020a), Canada (Denault et al., 2009), Lesotho (D'Isidoro et al., 2020), Finland (Solomon et al., 2020), Argentina (Genchi et al., 2018), Britain (Bett and Thornton, 2016), Saudi Arabia (Sahin, 2000) and Chile (Odeh and Watts, 2019). It has also been used at the international level (e.g. Latin America (Viviescas et al., 2019), Iberian Peninsula (Gutiérrez et al., 2017)), and even at intercontinental level (e.g. Canada vs. Noruega (Denault et al., 2009) and 
Europe vs. Africa (Kougias et al., 2016)). Under this approach, there is a high complementarity between 0 two resources when their time series have a high negative correlation coefficient, sometimes called anti-correlation (e.g. Bett and Thornton, 2016; Cao et al., 2019; Kougias et al., 2016; Gutiérrez et al., 2017; Slusarewicz and Cohan, 2018). In our opinion, a complementarity metric should measure the regularity of the sum of all available resources, and correlation coefficients fail to do that. The use of correlation coefficients also has the following issues:

1. Negative correlation is not complementarity. Although complementarity entails correlation, correlation does not entail complementarity. For example, the correlation between speed and height in a pendulum is negative, but they are not complementary.

2. Dimensions matter. In the above example, speed and height have different units. Obviously, for two or more variables to be complementary, they must have the same dimension. If they have different dimensions, one can not sum them, and their complementarity has no sense. In the pendulum example above, the complementary variables are kinetic energy and potential energy.

3. The scale of the variables does matter. For example, streamflows from a large river and a small creek could exhibit a sizeable negative correlation, but the large one dominates their sum, and hence the complementarity value should be low.

4. Linearity of the relation is also an issue. Even if correlation coefficients were suitable for assessing complementarity, the selection of Pearson's, Kendall's, Spearman's or any other coefficient should be done on the basis of some physical or mathematical considerations.

Besides correlation, among the other methods used to evaluate complementarity, one can mention the one proposed by Beluco et al. (2008) as 0 the product of three partial indices: (i) partial time complementarity index, which evaluates the time interval between the minimum values of two sources; (ii) partial energy complementarity index, which evaluates the relationship between the average values of two sources; (iii) partial amplitude-complementarity index, which assesses the differences between maximum and minimum values of the two energy sources. This idea works well for simple annual sinusoidal series but fails for more complicated series. Borba and Brito (2017) proposed the dimensionless ratio between the actual generation discarding excess power and the average generation. This index is more a measure of volatility than complementarity. Han et al. (2019) presented two indices of the complementary rate of fluctuation (CROF) and complementary rate of the ramp (CROR) to analyze the complementary degree of sequences with different fluctuation characteristics. Their rate of fluctuation seems to follow the concept of total variation that we present. Neto et al. (2020) used the concept of Daily Physical Guarantee (DPG), similar to firm power, to evaluate different renewable energy source's complementarity. The general idea is interesting but depends on the system's particular characteristics and does not provide a direct complementarity metric. Canales et al. (2020a); Arias et al. (2015); Canales et al. (2020b) introduced a method that assesses temporal complementarity between three variable energy sources, using a combination of correlation coefficients, Euclidean vectors, compromise programming, and normalization. The method's basis is correlation analysis, and therefore their proposal shares the criticism we made above. For a complete review of different methods to evaluate complementarity see Jurasz et al. (2020). We think that none of those proposals is a satisfactory way of measuring complementarity.

Looking to solve these issues, we propose two novel indices based on the Total Variation theory, described in section 2. To illustrate the use and properties of the new indices, we apply them to four cases of study (section 3): 1) two autoregresive processes; 2) wind vs. hydro in the Colombian electricity market; 3) two hydropower sources in the Colombian electricity market; and 4) fifteen hydropower sources in the Colombian electricity market. 


\section{Method}

In ordinary language, a complement of something is a thing that completes or brings perfection. In math, two numbers complement each other when their sum is constant, usually 1 or 100 . A measure of complementarity or complementariness between two functions should assess the perfection of their sum. For perfection here, we will take the regularity of the sum. Therefore, the measure of complementarity is related to the measure of regularity. A satisfactory way of measuring the regularity of a function is through its total variation, which allows for simple discontinuities and has more satisfactory properties than the variance (Kolmogorov and Fomin, 1970, p. 328) (Lasota and Mackey, 1994, p. 140).

In section 2.1 we review the concept and main mathematical properties of Total Variation. Then, we propose two indices, $\phi$ and $\phi^{\prime}$, as novel metrics of complementarity (sections 2.2 and 2.3). Afterwards, we present one theoretical and two real case studies to evaluate the suitability of $\phi$ and $\phi^{\prime}$ to quantify complementarity: 1) two $\mathrm{AR}(1)$ processes (section 3.1), 2) daily wind and hydropower generation time series in Colombia (section 3.2), and 3) the daily inflows to two hydropower reservoirs in Colombia (section 3.3). Results are compared between $\phi$ and $\phi^{\prime}$. In section 3.4 we present the application of $\phi$ index for the complementarity evaluation of multiple time series.

\subsection{Total variation}

According to Kolmogorov and Fomin (1970); Lasota and Mackey (1994), the total variation of a function $f$ defined on an interval $[a, b]$ is

$$
\bigvee_{a}^{b} f=\sup \sum_{i=1}^{n}\left|f\left(t_{i}\right)-f\left(t_{i-1}\right)\right|,
$$

where the supremum is taken over all possible finite partitions $a=t_{1}<\ldots<t_{n}=b$ of $[a, b]$.

If the sum is bonded, $f$ is said to be of bounded variation on $[a, b]$. Some important properties of the total variation are worth recalling:

1. The total variation of a constant function is zero, and conversely, if $\bigvee_{a}^{b} f=0$ then $f$ is constant on $[a, b]$
2. The total variation of a monotonic function $f$ on $[a, b]$ is $|f(b)-f(a)|$

3. $\bigvee_{a}^{b}(\alpha f)=|\alpha| \bigvee_{a}^{b}(f)$ for any constant $\alpha$.

4. If $f_{1}$ and $f_{2}$ are functions of bounded variation then so is $f_{1}+f_{2}$, and

$$
\bigvee_{a}^{b}\left(f_{1}+f_{2}\right) \leq \bigvee_{a}^{b}\left(f_{1}\right)+\bigvee_{a}^{b}\left(f_{2}\right)
$$

5. If $a<b<c$ then

$$
\bigvee_{a}^{c}(f)=\bigvee_{a}^{b}(f)+\bigvee_{b}^{c}(f) .
$$

From the above properties it is clear that the total variation of a function $f$ with values $f\left(t_{k}\right)$ at the points $a=t_{0}<t_{1}<\ldots<t_{n}=b$, and linear interpolation in between the given points is

$$
\bigvee_{a}^{b}(f)=\sum_{k=0}^{n-2}\left|f\left(t_{k+1}\right)-f\left(t_{k}\right)\right| .
$$

This property is useful to compute the total variation of a time series.

To illustrate, consider the following sequence of functions $g_{n}(t)=\sin (2 \pi n t), n \geq 1$. Clearly, in $[0,1]$ all the functions have 0 mean and variance $1 / 2$, but $\bigvee_{0}^{1}\left(g_{n}\right)=4 n$ Lasota and Mackey (1994).

Taking into account the above, it is possible to define a complementarity index.

\subsection{Total Variation Complementarity Index}

Given two functions $f_{1}(t)$ and $f_{2}(t)$, not both constant, their complementarity index $\phi\left(f_{1}, f_{2}\right)$ over the time interval $[a, b]$ is defined as

$$
\phi\left(f_{1}, f_{2}\right)=1-\frac{\bigvee_{a}^{b}\left(f_{1}+f_{2}\right)}{\bigvee_{a}^{b}\left(f_{1}\right)+\bigvee_{a}^{b}\left(f_{2}\right)} .
$$

Clearly, $0 \leq \phi\left(f_{1}, f_{2}\right) \leq 1$, and when $\phi\left(f_{1}, f_{2}\right)=$ 1 there is perfect complementary between $f_{1}$ and $f_{2}$, for in that case $f_{1}+f_{2}$ is a constant. The generalization to any number of functions is direct.

As an especial case, consider the Total Variation Complementarity index between the series of anomalies. Clearly, the total variation 
complementarity $\phi$ between the series $f_{1}, f_{2}, \ldots, f_{n}, \quad$ each function $\phi\left(f_{1}, \ldots, f_{n}\right)=\phi\left(f_{1}-\mu_{1}, \ldots, f_{n}-\right.$ is equal to the complementarity between the series $205 \mu_{n}$ ), because

of anomalies with respect to a constant mean for

$$
\frac{\bigvee_{a}^{b}\left(f_{1}+f_{2}+\ldots+f_{n}\right)}{\bigvee_{a}^{b}\left(f_{1}\right)+\bigvee_{a}^{b}\left(f_{2}\right)+\ldots+\bigvee_{a}^{b}\left(f_{n}\right)}=\frac{\bigvee_{a}^{b}\left[\left(f_{1}-\mu_{1}\right)+\left(f_{2}-\mu_{2}\right)+\ldots+\left(f_{n}-\mu_{n}\right)\right]}{\bigvee_{a}^{b}\left(f_{1}-\mu_{1}\right)+\bigvee_{a}^{b}\left(f_{2}-\mu_{2}\right)+\ldots+\bigvee_{a}^{b}\left(f_{n}-\mu_{n}\right)}
$$

\section{Summary of the $\phi$ index characteristics}

1. If $f_{1}$ is the vertical reflection of $f_{2},\left(f_{1}+f_{2}\right)$ is a constant, then $\bigvee_{t_{a}}^{t_{b}}\left(f_{1}+f_{2}\right)=0$ and $\phi=1$.

2. $0 \leq \phi \leq 1$. For the extreme cases $\phi=1$ means that there is perfect complementarity and for $\phi=0$ there is no complementarity.

3. $\phi$ is symmetric.

4. The $\phi$ index presents the same result when is evaluated on anomaly series compared to their corresponding pure series.

5. The $\phi$ index could be applied to two or more series.

\subsection{Variance Complementarity Index}

If one wants to stay within the correlation concept, 220 it is possible to correct the use of $\rho$ as a metric for complementarity to consider the variables' scale. For that, considering Eq. 5, one can replace total variation by variance. This is, given two functions $f_{1}(t)$ and $f_{2}(t)$, not both constant, their variance complementarity index $\phi_{v}\left(f_{1}, f_{2}\right)$ is

$$
\phi_{v}\left(f_{1}, f_{2}\right)=1-\frac{\operatorname{Var}\left[f_{1}+f_{2}\right]}{\operatorname{Var}\left[f_{1}\right]+\operatorname{Var}\left[f_{2}\right]},
$$

which simplifies to

$$
\begin{aligned}
\phi_{v}\left(f_{1}, f_{2}\right) & =-\frac{2 \operatorname{Cov}\left[f_{1}, f_{2}\right]}{\operatorname{Var}\left[f_{1}\right]+\operatorname{Var}\left[f_{2}\right]} \\
& =-\rho_{1,2} \frac{2 \sqrt{\operatorname{Var}\left[f_{1}\right] \operatorname{Var}\left[f_{2}\right]}}{\operatorname{Var}\left[f_{1}\right]+\operatorname{Var}\left[f_{2}\right]} .
\end{aligned}
$$

Notice that $\phi_{v}$ so defined is the negative of the correlation coefficient times the ratio of the geometric and arithmetic means of the variances. This last factor is always less or equal to one, with equality when the two variances are equal. Therefore, $\phi_{v}\left(f_{1}, f_{2}\right)$ varies between -1 and 1 . It is convenient to re-scale it to the range zero to one by means of

$$
\phi^{\prime}\left(f_{1}, f_{2}\right)=\left(\phi_{v}+1\right) / 2 .
$$

\section{Summary of the $\phi^{\prime}$ index characteristics}

1. If $f_{1}$ is the vertical reflection of $f_{2}, \operatorname{Var}\left[f_{1}\right]=$ $\operatorname{Var}\left[f_{2}\right]$, then $\rho_{1,2}=-1, \phi_{v}=1$ and $\phi^{\prime}=1$.

2. $0 \leq \phi^{\prime} \leq 1$. For the extreme cases $\phi^{\prime}=1$ means that there is perfect complementarity and for $\phi^{\prime}=0$ there is no complementarity.

3. $\phi^{\prime}$ is symmetric.

4. The $\phi^{\prime}$ index presents the same result when is evaluated on anomaly series compared to their corresponding pure series.

5. The $\phi^{\prime}$ index could be applied just to two series.

\section{Cases of study}

\subsection{Auto-regressive process}

Auto-regressive processes are a well-known time series method widely used for all sorts of applications (Box et al., 2011). This section shows an expression for the total variance complementarity index between two cross-correlated auto-regressive processes of order one. The purpose is to clarify the two ways of measuring complementarity, namely the total variation and the variance complementarity index. Let $\mathbf{z}_{t}$ be a vector time series with two components, $x_{t}$ and $y_{t}$, both with zero mean and unit variance. The multivariate auto-regressive process of order one is

$$
\mathbf{z}_{t+1}=\mathbf{A} \mathbf{z}_{t}+\mathbf{B} \mathbf{v}_{t+1},
$$

where the matrices $\mathbf{A}$ and $\mathbf{B}$ are constant parameters, and $\mathbf{v}_{t}$ is a vector time series of uncorrelated random variables with zero mean and 
unit variance. This is:

$$
\begin{aligned}
\mathrm{E}\left[\mathbf{v}_{t}\right] & =\mathbf{0}, \\
\operatorname{Var}\left[\mathbf{v}_{t}\right] & =\mathrm{E}\left[\mathbf{v}_{t} \mathbf{v}_{t}^{T}\right]=\mathbf{I}, \\
\operatorname{Cov}\left[\mathbf{v}_{t}, \mathbf{v}_{t+j}\right] & =\mathrm{E}\left[\mathbf{v}_{t} \mathbf{v}_{t+j}^{T}\right]=\mathbf{0},
\end{aligned}
$$

for all $j \neq 0$, and $\mathbf{I}$ the identity matrix. For $j>0$ the vector $\mathbf{v}_{t+j}$ is uncorrelated with $\mathbf{z}_{t}$,

$$
\operatorname{Cov}\left[\mathbf{z}_{t}, \mathbf{v}_{t+j}\right]=\mathrm{E}\left[\mathbf{z}_{t} \mathbf{v}_{t+j}^{T}\right]=\mathbf{0} \text { for } j>0 .
$$

The second order correlation structure of the process $\mathbf{z}_{t}$ depends on the matrices $\mathbf{M}_{0}=$ $\operatorname{Var}\left[\mathbf{z}_{t}\right]=\mathrm{E}\left[\mathbf{z}_{t} \mathbf{z}_{t}^{T}\right]$ and $\mathbf{M}_{1}=\operatorname{Cov}\left[\mathbf{z}_{t+1}, \mathbf{z}_{t}\right]=$ $\mathrm{E}\left[\mathbf{z}_{t+1} \mathbf{z}_{t}^{T}\right]$, with components

$$
\begin{aligned}
& \mathbf{M}_{0}=\left(\begin{array}{cc}
\operatorname{Var}\left[x_{t}\right] & \operatorname{Cov}\left[x_{t}, y_{t}\right] \\
\operatorname{Cov}\left[y_{t}, x_{t}\right] & \operatorname{Var}\left[y_{t}\right]
\end{array}\right) \\
& \mathbf{M}_{0}=\left(\begin{array}{cc}
1 & \rho_{x y} \\
\rho_{x y} & 1
\end{array}\right),
\end{aligned}
$$

that is symmetric and $t$-independent. Whereas

$$
\begin{aligned}
& \mathbf{M}_{1}=\left(\begin{array}{cc}
\operatorname{Cov}\left[x_{t+1}, x_{t}\right] & \operatorname{Cov}\left[x_{t+1}, y_{t}\right] \\
\operatorname{Cov}\left[y_{t+1}, x_{t}\right] & \operatorname{Cov}\left[y_{t+1}, y_{t}\right]
\end{array}\right) \\
& \mathbf{M}_{1}=\left(\begin{array}{cc}
\rho_{x} & \rho_{x_{t+1}, y_{t}} \\
\rho_{y_{t+1}, x_{t}} & \rho_{y}
\end{array}\right)
\end{aligned}
$$

is not symmetric.

To find expressions for the parametric matrices post-multiply eq. (10) by $\mathbf{z}_{t}$ and take the expected value

$$
\mathrm{M}_{1}=\mathrm{AM}_{\mathbf{0}}
$$

therefore

$$
\mathrm{A}=\mathrm{M}_{1} \mathrm{M}_{0}^{-1} .
$$

Similarly, post-multiply eq. (10) by $\mathbf{z}_{t+1}$ and take expected values to get

$$
\mathrm{M}_{0}=\mathrm{M}_{1} \mathrm{M}_{0}^{-1} \mathrm{M}_{1}^{\mathrm{T}}+\mathrm{BB}^{\mathrm{T}} .
$$

One can then obtain B from Eq. 17 assuming it is triangular. A simple way is to assume that $\rho_{x_{t+1}, y_{t}}=\rho_{x y} \rho_{x}$ and $\rho_{y_{t+1}, x_{t}}=\rho_{x y} \rho_{y}$ (Matalas, 1967). In that case, $\mathbf{M}_{\mathbf{1}}=\mathbf{D M}_{\mathbf{0}}$, with $\mathbf{D}$ a diagonal

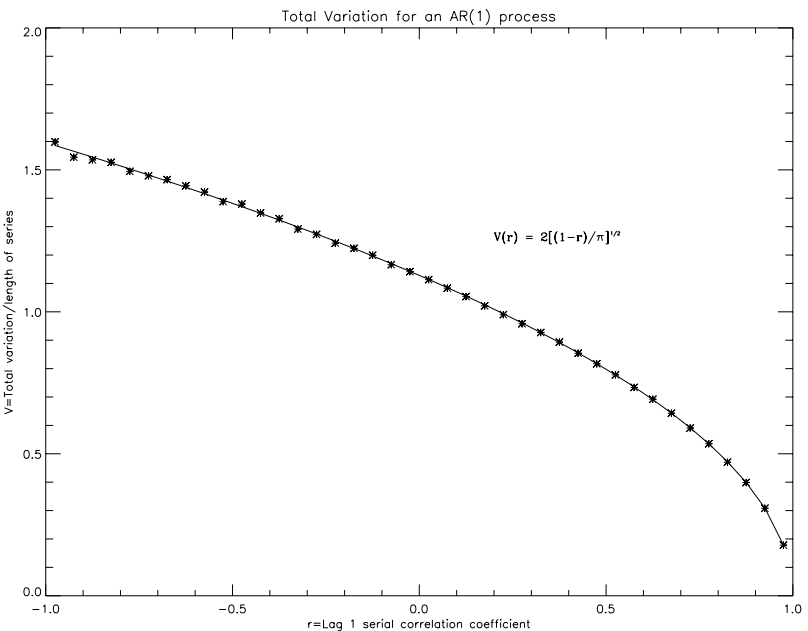

Figure 1. Expected value of the total variation of an univariate auto-regressive process as a function of the lag-one auto-correlation coefficient. The points are the average of 100 simulations. The continuous line is eq. (21).

matrix

$$
\mathbf{D}=\left(\begin{array}{cc}
\rho_{x} & 0 \\
0 & \rho_{y}
\end{array}\right)
$$

Therefore eq. (16) is now

$$
\mathrm{A}=\mathrm{M}_{1} \mathrm{M}_{0}^{-1}=\mathrm{D} .
$$

Similarly, eq. (17) simplifies to

$$
\begin{aligned}
\mathbf{B B}^{\mathbf{T}} & =\mathbf{M}_{\mathbf{0}}-\mathbf{D M}_{\mathbf{0}} \mathbf{D} \\
\mathbf{B B}^{\mathbf{T}} & =\left(\begin{array}{cc}
1-\rho_{x}^{2} & \rho_{x y}\left(1-\rho_{x} \rho_{y}\right) \\
\rho_{x y}\left(1-\rho_{x} \rho_{y}\right) & 1-\rho_{y}^{2}
\end{array}\right)
\end{aligned}
$$

that one can solve for $\mathbf{B}$

$$
\mathbf{B}=\left(\begin{array}{cc}
\sqrt{1-\rho_{x}^{2}} & 0 \\
\frac{\rho_{x y}\left(1-\rho_{x} \rho_{y}\right)}{\sqrt{1-\rho_{x}^{2}}} & \sqrt{1-\rho_{y}^{2}-\frac{\rho_{x y}^{2}\left(1-\rho_{x} \rho_{y}\right)^{2}}{1-\rho_{x}^{2}}}
\end{array}\right) .
$$

The expected value of the total variation of an univariate auto-regressive process (the first component of eq. (10)) is

$$
\mathrm{E}\left[\bigvee_{1}^{n} x_{t}\right]=(n-1) \times \frac{2}{\sqrt{\pi}} \sqrt{1-\rho_{x}}
$$

Fig. 1 shows the expected value as a function of the lag-one autocorrelation coefficient $\rho_{x}$.

The result in eq. (21) comes realizing that $\mid x_{t+1}-$ $x_{t}|=| \sqrt{1-\rho^{2}} v_{t+1}-(1-\rho) x_{t} \mid$, where both $x_{t}$ and $v_{t}$ are independent random variables, with 

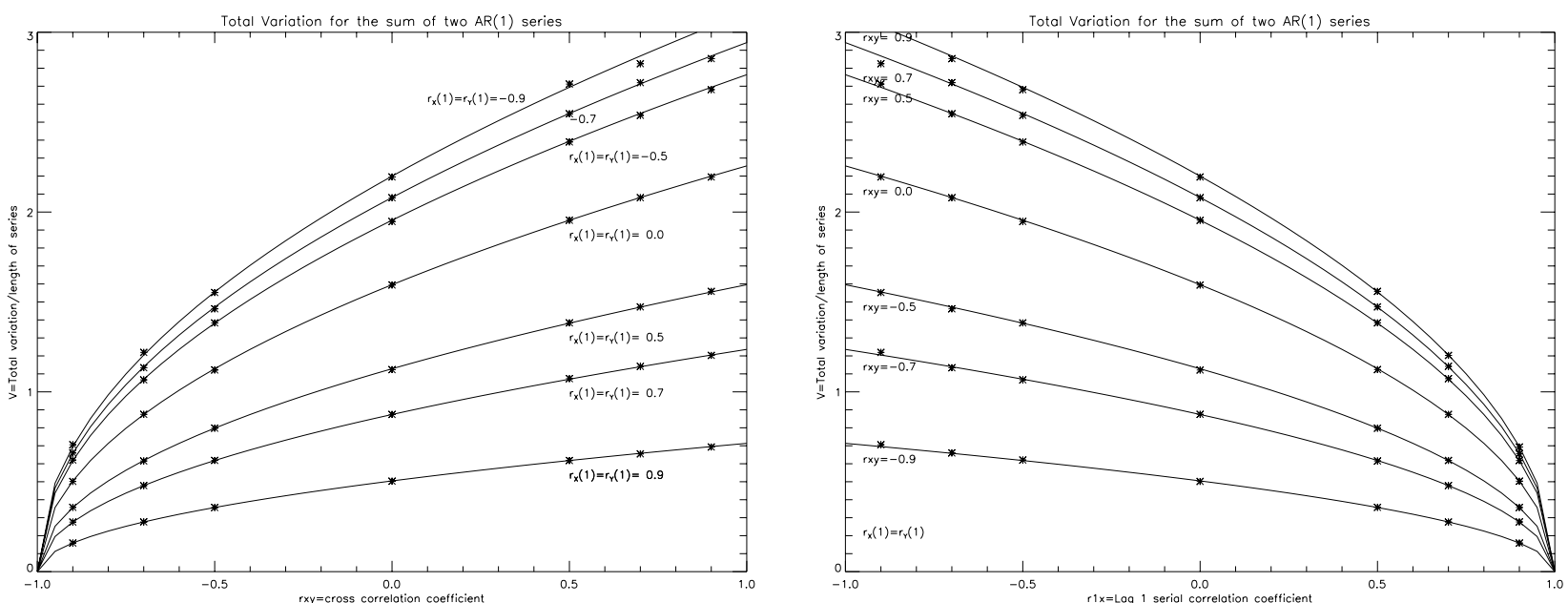

Figure 2. Expected value of the total variation of the sum of two AR(1) series as a function of $\rho_{x y}$ for different values of $\rho_{y}=-\rho_{x}$ (left panel), and as a function of $\rho_{y}=-\rho_{x}$ for different vales of $\rho_{x y}$ (right panel). Points are average of 100 simulations and the continuous line is eq. (24).

zero mean and unit variance. In addition, one usually assumes that their distribution is Gaussian. Therefore, the expected value of $\bigvee_{1}^{n}\left(x_{t}\right)$ is

$$
\begin{aligned}
& \mathrm{E}\left[\begin{array}{l}
n \\
1
\end{array} x_{t}\right]=\mathrm{E}\left[\sum_{k=0}^{n-2}\left|x_{t+1}-x_{t}\right|\right] \\
& \mathrm{E}\left[\begin{array}{l}
n \\
1
\end{array} x_{t}\right]=\sum_{k=0}^{n-2} \mathrm{E}\left[\left|\sqrt{1-\rho^{2}} v_{t+1}-(1-\rho) x_{t}\right|\right] .
\end{aligned}
$$

A simple calculation of the expected value in eq. (22) gives eq. (21).

Similar arguments apply to the calculation of the expected value of the total variation of the sum $x_{t}+$ $285 y_{t}$ :

$$
\mathrm{E}\left[\bigvee_{1}^{n}\left(x_{t}+y_{t}\right)\right]=\sum_{k=0}^{n-2} \mathrm{E}\left[\left|\left(b_{1,1}+b_{2,1}\right) v_{t+1}+b_{2,2} w_{t+1}-\left(1-a_{1,1}\right) x_{t}-\left(1-a_{2,2}\right) y_{t}\right|\right]
$$

where the $a_{i, j}$ and $b_{i, j}$ are respectively the components of the matrices $\mathbf{A}$ and $\mathbf{B}$ in eqs. (18) and (20). All the random variables in eq. (23) 90 are Gaussian, with zero mean and unit variance. $v_{t+1}$ and $w_{t+1}$ are mutually independent and independent of $x_{t}, y_{t}$. The covariance between $x_{t}$ and $y_{t}$ is $\rho_{x y}$. Therefore, using properties of the Gaussian distribution, the absolute value in eq. (23) corresponds to a Gaussian distribution with zero mean and variance equal to $\left(b_{1,1}+b_{2,1}\right)^{2}+b_{2,2}^{2}+$ $\left(1-a_{1,1}\right)^{2}+\left(1-a_{2,2}\right)^{2}+2\left(1-a_{1,1}\right)\left(1-a_{2,2}\right) \rho_{x y}=$ $2\left(1+\rho_{x y}\right)\left(2-\rho_{x}-\rho_{y}\right)$.

Finally, the expected value of the total variation 300 of the sum is

$$
\mathrm{E}\left[\mathrm{V}_{1}^{n}\left(x_{t}+y_{t}\right)\right]=(n-1) \frac{2}{\sqrt{\pi}} \sqrt{\left(1+\rho_{x y}\right)\left(2-\rho_{x}-\rho_{y}\right)}
$$

Figure 2 illustrates this result.

Using the above results, eqs. (21) and (24), the total variation complementarity index between two auto-regressive time series with lag-one correlation coefficients $\rho_{x}$ and $\rho_{y}$, and cross correlation $\rho_{x y}$, is

$$
\begin{aligned}
\phi\left(x_{t}, y_{t}\right) & =1-\frac{\bigvee_{1}^{n}\left(x_{t}+y_{t}\right)}{\bigvee_{1}^{n}\left(x_{t}\right)+\bigvee_{1}^{n}\left(y_{t}\right)} \\
& =1-\frac{\sqrt{\left(1+\rho_{x y}\right)\left(2-\rho_{x}-\rho_{y}\right)}}{\sqrt{1-\rho_{x}}+\sqrt{1-\rho_{y}}} .
\end{aligned}
$$

Figure 3 illustrate some particular cases of this result. We are interested in the comparison of $\phi$ with $\phi^{\prime}$. Recall that the unit variance of $x_{t}$ and $y_{t}$ implies that $\phi^{\prime}\left(x_{t}, y_{t}\right)=\left(1-\rho_{x y}\right) / 2$. 

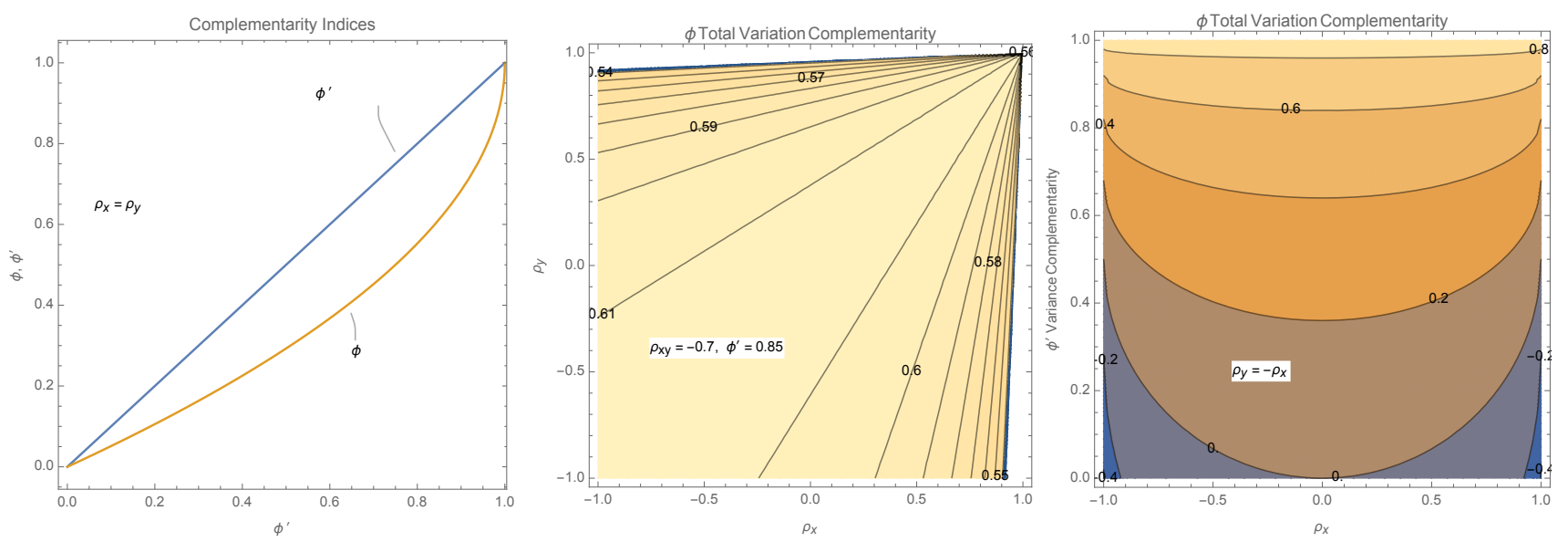

Figure 3. Expected value of total variation complementarity index as a function of $\rho_{x}, \rho_{y}$ and $\phi^{\prime}$ for the sum of two AR(1) series: left panel, $\rho_{y}=\rho_{x}$; center panel, $\rho_{x y}=-0.7, \phi^{\prime}=0.85$; and right panel, $\rho_{y}=-\rho_{x}$. Notice a region of negative values that correspond to non-admissible parameter values for the $\operatorname{AR}(1)$ process.

A first observation is that for $\rho_{x y}=-1$ both $\phi$ and $\phi^{\prime}$ are one, that is perfect complementarity. If $\rho_{x}=\rho_{y}$ the total variation complementarity is independent of $\rho_{x}$, with $\phi=1-\sqrt{2\left(1+\rho_{x y}\right)} / 2 \leq$ $\phi^{\prime}=\left(1-\rho_{x y}\right) / 2$ (left panel of fig. 3). Still within the equality of the lag-one correlation coefficients, the case $\rho_{x y}=0$ and therefore $\phi=1-\sqrt{2} / 2 \approx$ 0.29 is interesting in comparison with $\phi^{\prime}=0.5$. That high a variance complementarity index in the absence of cross-correlation may come from how the range of the index was put between zero and one in eq. (9). In fact, positive cross correlations give $0<\phi^{\prime}<1 / 2$, something that seems high. An alternative to eq. (9) could be $1-\rho_{x y}$ for negative correlations and zero for positive ones. We think that both alternatives are inferior than the total variation complementarity index. For null cross correlation, of the order of $30 \%$ of the total variation of the sum cancels out just by chance, whereas either $50 \%$ or $0 \%$ seem off.

For constant $\rho_{x y}=-0.7$ in the center panel of fig. 3 , the total variation complementarity does depend on both $\rho_{x}$ and $\rho_{y}$ although $\phi^{\prime}=0.85$.

For the case $\rho_{x}=-\rho_{y}$ (right panel of fig. 3) $\phi$ depends on $\rho_{x}$ except for $\phi^{\prime}$ close to one. In all cases $\phi$ is less than $\phi^{\prime}$.

We conclude from this comparison that the total variation complementarity index has more information than the variance complementarity.

\subsection{Case of study 2: wind vs. hydro in the Colombian electricity market}

The total installed capacity for electricity generation in Colombia is approximately $17.5 \mathrm{GW}$, of which $68.3 \%$ is hydro while the only one wind plant accounts for near $0.1 \%$. In fact, this big scale difference impacts the complementarity assessment.

To quantify the complementarity between wind and hydro-resources in the Colombian electricity system, we use daily time series provided by $\mathrm{XM}$, the electric system administrator and operator in Colombia. For hydro-resources, we analyze natural inflows (in units of energy) to the largest 15 hydropower reservoirs, with a total effective installed capacity of $7,668 \mathrm{MW}(\approx 70 \%$ of total hydro-capacity) and records from 2000 to 2020. These rivers are San Lorenzo, Nare, Guatapé, San Carlos, Tenche, Guadalupe, Grande, Porce II, Miel I, Urrá, Betania, Batá, Guavio, Alto Anchicayá, and Salvajina. For details on the hydropower system of Colombia, see XM the internet site. For wind resources, we analyze daily wind power generation from the Jepirachi plant, with 19.5 MW of effective installed capacity and records for the period 20042020. Notice that physical dimensions of both series are the same (energy).

Figure 4 shows the estimation of $\phi$ and $\phi^{\prime}$ indices to the daily series and the annual cycles of hydroinflows and wind generation with moving averages of 31 days (top panel). Considering the large scale 

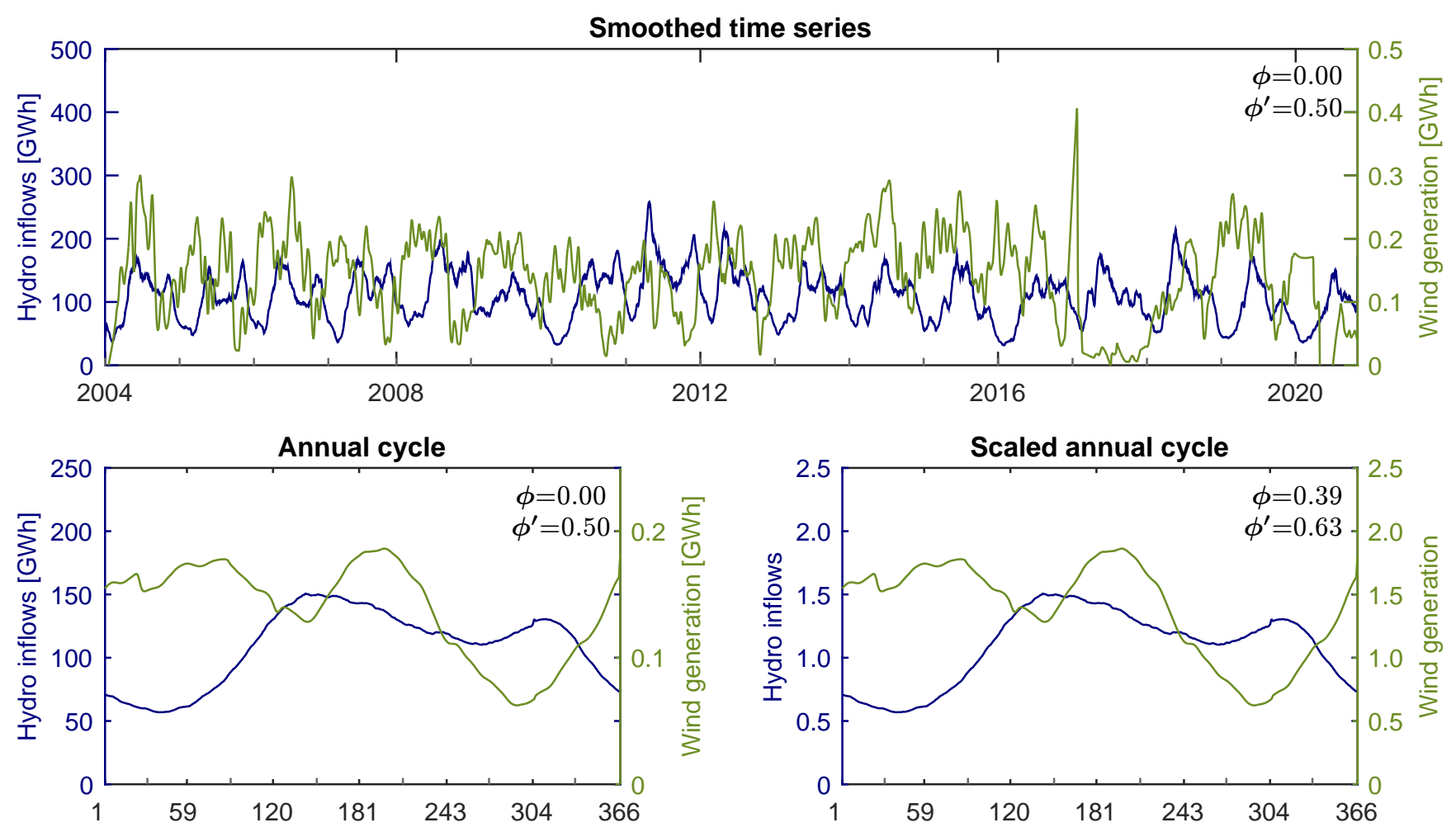

Figure 4. Comparison of $\phi$ and $\phi^{\prime}$ indices between wind and hydraulic resources in Colombia electricity market, with smoothed moving averages of 31 days. The scale factor in the case of scaled annual cycle is 1/100 for hydro-inflow series and $1 / 10$ for wind-power generation series.

difference of the accumulated hydro-inflows and the Jepirachi plant, the scaled series's complementarity was also studied (bottom panel), being the scale factor $1 / 100$ for hydro-inflow series and 1/10 for wind generation series. Table 1 presents the results for all analyzed cases.

As expected, because of the scale differences, $\phi$ does not show complementarity, while $\phi^{\prime}$ index indicates a complementarity of $50 \%$ for the daily and annual cycles. When one uses the scaled series, the $\phi$ index increases to $39 \%$. We interpret this scaled analysis to evaluate the resources' potential complementarity if the resources' scale were comparable.

The difference in the results may come because 380 the $\phi$ index depends on each series's serial correlation, while $\phi^{\prime}$ does not. Besides, there is a tendency of $\phi^{\prime}$ to exceed $\phi$, overestimating the complementarity quantification.

\subsection{Case of study 3: two hydropower sources in the Colombian electricity market}

Nare and Guavio are two important rivers of the hydropower reservoir system of Colombia. The Andes mountain range separates the two basins, causing important differences in their hydroclimatic patterns. For instance, seasonality of rainfall is unimodal in the Guavio basin and bimodal in the Nare basin (Urrea et al., 2019), and streamflows show these regimes too (Fig. 5). According to XM, these rivers belong to different hydrological regions (see details in the XM website).

We computed the complementarity indices between the daily natural inflows (in energy units) of Nare and Guavio rivers for the period 2000-2020. Figure 5 shows the results, where the left panel shows the 31-day moving average daily inflows and the right panel the annual cycle at daily time-step for both rivers. Table 2 lists the results for all analyzed cases. 
Table 1. Complementarity between wind and hydro resources in Colombia. The daily time series were smoothed, obtaining moving averages of 31 days. Variance has units of $G W h^{2}$ in the case of smoothed time series and annual cycle. In all other cases, the variables are dimensionless. $\rho_{1}$ represents the exponential value of the slope of the linear regression for the autocorrelograms of both resources.

\begin{tabular}{llllllll}
\hline Group & $\sigma_{\text {Hydro }}^{2}$ & $\rho_{1 \text { Hydro }}$ & $\sigma_{\text {Wind }}^{2}$ & $\rho_{\text {1Wind }}$ & $\rho_{\text {Hydro,Wind }}$ & $\phi$ & $\phi^{\prime}$ \\
\hline Smoothed time series & 1,508 & 0.98 & 0.01 & 0.98 & -0.12 & 0.00 & 0.50 \\
\hline Annual cycle & 878 & - & 0.00 & - & -0.26 & 0.00 & 0.50 \\
\hline Scaled series & 0.09 & - & 0.13 & - & -0.26 & 0.39 & 0.63 \\
\hline
\end{tabular}

Table 2. Complementarity between 31-day moving average daily streamflows of Nare and Guavio rivers. Variance has units of $G W h^{2}$ in the case of smoothed time series and annual cycle. In all other cases, the variables are dimensionless. $\rho_{1}$ represents the linear regression slope for the auto-correlograms of both resources.

\begin{tabular}{llllllll}
\hline Group & $\sigma_{\text {Nare }}^{2}$ & $\rho_{1 \text { Nare }}$ & $\sigma_{\text {Guavio }}^{2}$ & $\rho_{1 \text { Guavio }}$ & $\rho_{\text {Nare,Guavio }}$ & $\phi$ & $\phi^{\prime}$ \\
\hline Smoothed time series & 62 & 0.98 & 129 & 0.98 & 0.25 & 0.20 & 0.38 \\
\hline Annual cycle & 17 & - & 101 & - & 0.49 & 0.18 & 0.33 \\
\hline Scaled series & 0.46 & - & 1.45 & - & 0.49 & 0.19 & 0.29 \\
\hline
\end{tabular}

The scale of both resources is not as different as in the previous case. Consequently, the $\phi$ index is similar for the daily and scaled series with values close to $20 \%$. Using the equivalent to eq. (25) that considers the values of variance (not shown), with the parameters contained in table 2 , one gets $\phi=$ 0.20 . The same value that one obtains using the time series and eqs. (4) and (5). This correspondence suggests that the auto-regressive model is good to estimate complementarity. Again, $\phi^{\prime}$ shows a higher result than $\phi$ for all cases, quantifying the complementarity around $38 \%$. The pattern is that the variance complementarity index tends to overestimate complementarity.

\subsection{Case of study 4: Hydropower integrated sources in the Colombian electricity market}

One of the main advantages of the $\phi$ index is that one can evaluate the complementarity not just between two but among any number of series. To illustrate this capability, we quantify the complementarity among the 15 largest rivers energy inflows in the Colombian hydroelectric system (described in section 3.2). These rivers are in different Colombia regions with different
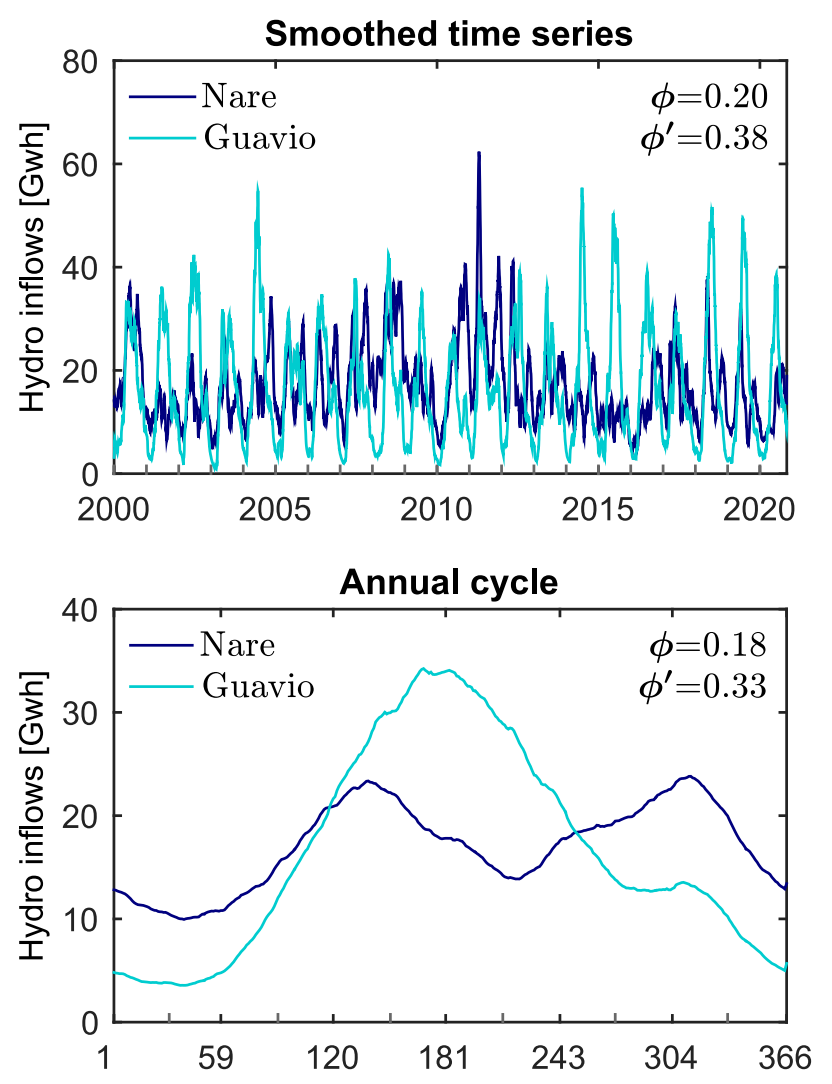

Figure 5. Assessment of complementarity by the $\phi$ and $\phi^{\prime}$ indices between the Nare and Guavio rivers. Left panel: 31day moving average daily inflows. Right panel: annual cycle at daily time-step. 

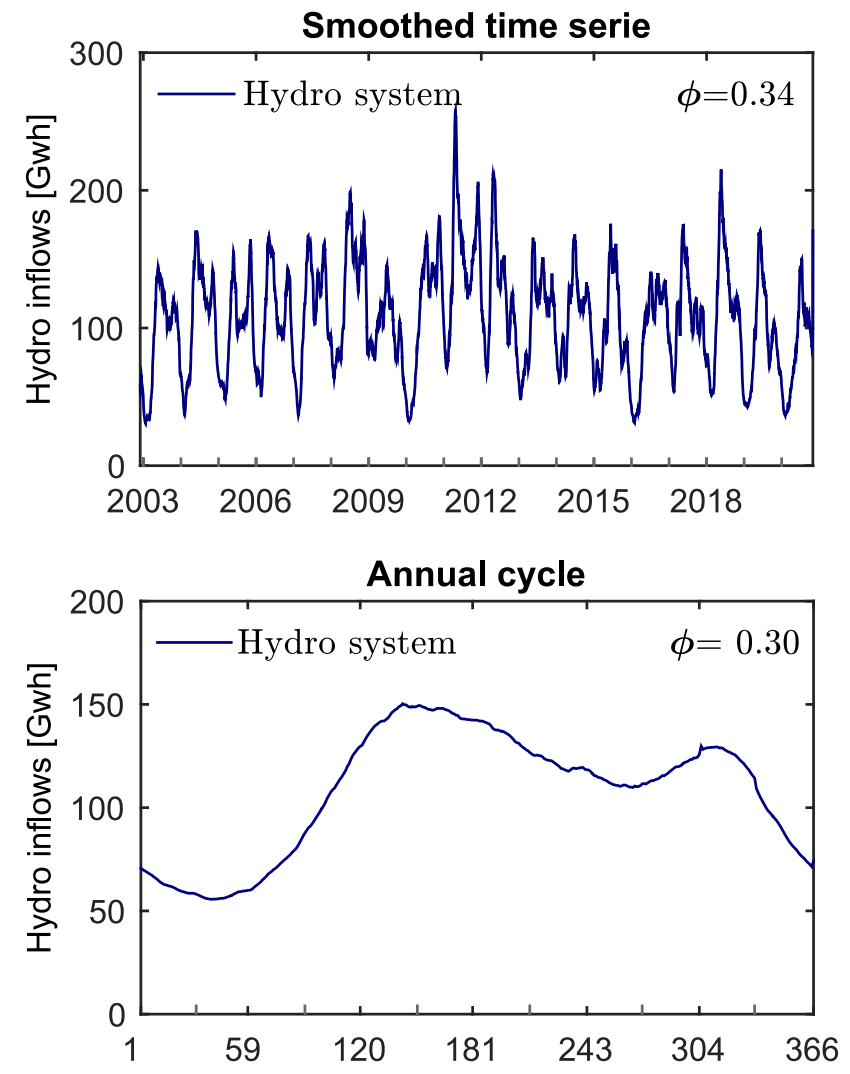

Figure 6. Quantification of the complementarity with $\phi 470$ Index among the energy inflows of the main 15 rivers of the Colombian electrical system, with smoothed moving averages of 31 days.

430 geographic and climatic characteristics, making the complementarity analysis relevant.

Figure 6 present the daily series and annual cycle of the integrated hydro system with moving averages of 31 days. The $\phi$ index shows a 5 complementarity of $34 \%$ for the smoothed series and $30 \%$ for the annual cycles. For the unsmoothed series (not shown), the complementarity is somewhat higher, reaching $38 \%$ and $46 \%$, respectively.

\section{Conclusions}

Correlation coefficients between time series are the common practice to evaluate and quantify complementarity. Pearson's correlation coefficient, which is the most frequently used metric of complementarity, has several fundamental issues that undermine its usefulness for that purpose. As it is scaled independent, one could use it for nondimensionally homogeneous variables, which does not make any sense. Also, if the series's scale is very different, the correlation coefficient may mislead the real complementarity. We showed that negative correlation is a necessary but not sufficient condition to uphold complementarity, demonstrated that scale has a large effect on complementarity magnitude. Also, that dimensional homogeneity between the variables is a requisite to provide physical sense to the concept of complementarity.

We propose a novel method based on the concept and main mathematical properties of Total Variation. The total variation complementarity index $(\phi)$ is a consistent and satisfactory metric for complementarity between any number of series. Index $\phi$ measures the sum of the variables' regularity and is unaffected by the issues pointed out 5 regarding correlation coefficients. Some essential properties of $\phi$ are: 1) $\phi$ is sensitive to the scale of the variables; 2) $\phi$ works only with dimensionally homogeneous variables; 3) $\phi$ can be used with any number of variables.

Given the unreliability of renewable energy sources, consistent evaluation of complementarity is a critical issue. Our proposed index fulfills this need, making a substantial contribution to energy planning.

Inspired by the total variation complementarity index, we proposed the variance complementarity index to correct the issues identified about Pearson's correlation coefficient in complementarity quantification.

We obtained analytical results for the expected value of two cross-correlated auto-regressive series, demonstrating that the variance complementarity index does not capture all the complementarity's dependencies. In particular, the example shows that 35 serial auto-correlation does affect complementarity. Besides, the applications to the Colombian power system show that the variance complementarity overestimates complementarity.

Finally, the proposed complementarity indices quantify the Colombian electricity system's complementarity due to its hydrological and climatic differences. The total variation complementarity index among the daily series of the hydro resources is $30 \%$. This figure is the first estimation of the 
complementarity among the 15 main rivers feeding the Colombian power system. The total variation complementarity index, $\phi$, between hydro and wind resources is almost null due to the wind component's negligible contribution. When one uses scaled variables, it is $39 \%$, representing the potential complementarity between these resources and could stimulate a higher level of investment in wind capacity development. The corresponding figures for the variance complementarity index between hydro and wind resources are $50 \%$ and $63 \%$. The $\phi$ index between Nare and Guavio is $20 \%$, whereas $\phi^{\prime}=38 \%$. These two rivers are often considered complementary because of their annual cycles. The indices put a solid basis and a concrete number on those intuitive ideas.

\section{Acknowledgments}

This work was supported by Minciencias, Universidad Nacional de Colombia, Universidad EIA and Interconexión Eléctrica S.A. under the grant contract number 80740-540-2020.

\section{References}

Arias, C., Ochoa, A., and Poveda, G. (2015). Influencia del fenómeno de El Niño - Oscilación del Sur sobre la leptospirosis humana en Colombia, 2008 - 2013. Biomédica 35, 115

Beluco, A., de Souza, P. K., and Krenzinger, A. (2008). A dimensionless index evaluating the time complementarity between solar and hydraulic energies. Renewable Energy 33, 2157-2165. doi:10.1016/j.renene.2008.01.019

Bett, P. E. and Thornton, H. E. (2016). The climatological relationships between wind and solar energy supply in Britain. Renewable Energy 87, 96-110. doi:10.1016/j.renene.2015.10.006

Borba, E. M. and Brito, R. M. (2017). An Index Assessing the Energetic Complementarity in Time between More than Two Energy Resources. Energy and Power Engineering 09, 505-514. doi:10.4236/epe.2017.99035

Box, G. E., Jenkins, G. M., and Reinsel, G. C. (2011). Time series analysis: forecasting and control, vol. 734 (John Wiley \& Sons)

Canales, F. A., Jurasz, J., Beluco, A., and Kies, A. (2020a). Assessing temporal complementarity between three variable energy sources through correlation and compromise programming. Energy 192, 116637. doi:10.1016/ j.energy.2019.116637

Canales, F. A., Jurasz, J., Kies, A., Beluco, A., Arrieta-Castro, M., and Peralta-Cayón, A. (2020b). Spatial representation of temporal complementarity between three variable energy sources using correlation coefficients and compromise programming. MethodsX 7, 100871. doi:10.1016/j.mex.2020.100871

Cantão, M. P., Bessa, M. R., Bettega, R., Detzel, D. H., and Lima, J. M. (2017). Evaluation of hydro-wind complementarity in the Brazilian territory by means of correlation maps. Renewable Energy 101, 1215-1225. doi:10.1016/j.renene.2016.10.012

Cantor, D., Ochoa, A., and Mesa, O. (2019). Complementariedad hidrológica en Colombia. In Día de la Energía 2019 (Envigado, Colombia: Universidad EIA). doi:10.13140/RG.2.2.10284. 82560

Cao, Y., Zhang, Y., Zhang, H., and Zhang, P. (2019). Complementarity assessment of windsolar energy sources in Shandong province based on NASA. The Journal of Engineering 2019, 4996-5000. doi:10.1049/joe.2018.9367

Castro, R. and Crispim, J. (2018). Variability and correlation of renewable energy sources in the Portuguese electrical system. Energy for Sustainable Development 42, 64-76. doi:10. 1016/j.esd.2017.10.005

de Oliveira Costa Souza Rosa, C., Costa, K., da Silva Christo, E., and Braga Bertahone, P. (2017). Complementarity of Hydro, Photovoltaic, and Wind Power in Rio de Janeiro State. Sustainability 9, 1130. doi:10.3390/su9071130

Denault, M., Dupuis, D., and Couture-Cardinal, S. (2009). Complementarity of hydro and wind power: Improving the risk profile of energy inflows. Energy Policy 37, 5376-5384. doi:10. 1016/j.enpol.2009.07.064

D’Isidoro, M., Briganti, G., Vitali, L., Righini, G., Adani, M., Guarnieri, G., et al. (2020). Estimation of solar and wind energy resources over Lesotho and their complementarity by means of WRF yearly simulation at high 
resolution. Renewable Energy 158, 114-129.635 doi:10.1016/j.renene.2020.05.106

dos Anjos, P. S., da Silva, A. S. A., Stošić, B., and Stošić, T. (2015). Long-term correlations and cross-correlations in wind speed and solar radiation temporal series from Fernando de Noronha Island, Brazil. Physica A: Statistical Mechanics and its Applications 424, 90-96. doi:10.1016/j.physa.2015.01.003

François, B., Borga, M., Anquetin, S., Creutin, J., Engeland, K., Favre, A. C., et al. (2014). Integrating hydropower and intermittent climaterelated renewable energies: a call for hydrology. Hydrological Processes 28, 5465-5468. doi:10. 1002/hyp.10274

Genchi, S. A., Vitale, A. J., Piccolo, M. C., and Perillo, G. M. (2018). Assessing wind, solar, and wave energy sources in the southwest of Buenos Aires province (Argentina). Investigaciones Geográficas 97. doi:10.14350/rig.59657

Gutiérrez, C., Gaertner, M. Á., Perpiñán, O., Gallardo, C., and Sánchez, E. (2017). A multi-step scheme for spatial analysis of solar and photovoltaic production variability and complementarity. Solar Energy 158, 100-116. doi:10.1016/j.solener.2017.09.037

Han, S., Zhang, L.-n., Liu, Y.-q., Zhang, H., Yan, J., Li, L., et al. (2019). Quantitative evaluation method for the complementarity of wind-solar-hydro power and optimization of wind-solar ratio. Applied Energy 236, 973-984. doi:10.1016/j.apenergy.2018.12.059

Henao, F., Viteri, J. P., Rodríguez, Y., Gómez, J., and Dyner, I. (2020). Annual and interannual complementarities of renewable energy sources in Colombia. Renewable and Sustainable Energy Reviews 134, 110318. doi:10.1016/j.rser.2020. 110318

Jurasz, J. (2017). Modeling and forecasting energy flow between national power grid and a solar-wind-pumped-hydroelectricity (PV-WT-PSH) energy source. Energy Conversion and Management 136, 382-394. doi:10.1016/j.enconman.2017.01.032

Jurasz, J., Canales, F., Kies, A., Guezgouz, M., and Beluco, A. (2020). A review on the complementarity of renewable energy sources: 6 Concept, metrics, application and future research directions. Solar Energy 195, 703-724. doi:10. 1016/j.solener.2019.11.087

Kay, M. (2015). Forecasting and Characterising Grid Connected Solar Energy and Developing Synergies with Wind Project results and lessons learnt (Australian Renewable Energy Agency)

Kolmogorov, A. and Fomin, S. (1970). Introduction Real Analysis Rev. English Ed (Dover)

Kougias, I., Szabó, S., Monforti-Ferrario, F., Huld, T., and Bódis, K. (2016). A methodology for optimization of the complementarity between small-hydropower plants and solar PV systems. Renewable Energy 87, 1023-1030. doi:10.1016/ j.renene.2015.09.073

Lasota, A. and Mackey, M. C. (1994). Chaos, Fractals, and Noise. Stochastic Aspects of Dynamics (Springer-Verlag New York), 2 edn. doi:10.1007/978-1-4612-4286-4

Li, H., Liu, P., Guo, S., Ming, B., Cheng, L., and Yang, Z. (2019). Long-term complementary operation of a large-scale hydro-photovoltaic hybrid power plant using explicit stochastic optimization. Applied Energy 238, 863-875. doi:10.1016/j.apenergy.2019.01.111

Matalas, N. C. (1967). Mathematical assessment of synthetic hydrology. Water Resources Research 3, 937-945

Monforti, F., Huld, T., Bódis, K., Vitali, L., D'Isidoro, M., and Lacal-Arántegui, R. (2014). Assessing complementarity of wind and solar resources for energy production in Italy. A Monte Carlo approach. Renewable Energy 63, 576-586. doi:10.1016/j.renene.2013.10.028

Moura, P. S. and de Almeida, A. T. (2010). Multiobjective optimization of a mixed renewable system with demand-side management. Renewable and Sustainable Energy Reviews 14, 1461-1468. doi:10.1016/j.rser.2010.01.004

Neto, P. B. L., Saavedra, O. R., and Oliveira, D. Q. (2020). The effect of complementarity between solar, wind and tidal energy in isolated hybrid microgrids. Renewable Energy 147, 339-355. doi:10.1016/j.renene.2019.08.134

Odeh, R. P. and Watts, D. (2019). Impacts of wind and solar spatial diversification on its market value: A case study of the Chilean electricity market. Renewable and Sustainable Energy 
Reviews 111, 442-461. doi:10.1016/j.rser.2019. 01.015

Parra, L., Gómez, S., Montoya, C., and Henao, F. (2020). Assessing the Complementarities of Colombia's Renewable Power Plants. Frontiers in Energy Research 8. doi:10.3389/fenrg.2020. 575240

Peña Gallardo, R., Medina Ríos, A., and Segundo Ramírez, J. (2020a). Analysis of the solar and wind energetic complementarity in Mexico. Journal of Cleaner Production 268, 122323. doi:10.1016/j.jclepro.2020.122323

Peña Gallardo, R., Ospino Castro, A., and Medina Ríos, A. (2020b). An Image ProcessingBased Method to Assess the Monthly Energetic Complementarity of Solar and Wind Energy in Colombia. Energies 13, 1033. doi:10.3390/ en13051033

Ren, G., Wan, J., Liu, J., and Yu, D. (2019). Spatial and temporal assessments of complementarity for renewable energy resources in China. Energy 177, 262-275. doi:10.1016/j.energy.2019.04.023

Sahin, A. Z. (2000). Applicability of WindSolar Thermal Hybrid Power Systems in the Northeastern Part of the Arabian Peninsula. Energy Sources 22, 845-850. doi:10.1080/ 009083100300001645

Schindler, D., Behr, H. D., and Jung, C. (2020). On the spatiotemporal variability and potential of complementarity of wind and solar resources. Energy Conversion and Management 218, 113016. doi:10.1016/j.enconman.2020. 113016

Silva, A. R., Pimenta, F. M., Assireu, A. T., and Spyrides, M. H. C. (2016). Complementarity of Brazil's hydro and offshore wind power. Renewable and Sustainable Energy Reviews 56, 413-427. doi:10.1016/j.rser.2015.11.045

Slusarewicz, J. H. and Cohan, D. S. (2018). Assessing solar and wind complementarity in Texas. Renewables: Wind, Water, and Solar 5, 7. doi:10.1186/s40807-018-0054-3

Solomon, A., Child, M., Caldera, U., and Breyer, C. (2020). Exploiting wind-solar resource complementarity to reduce energy storage need. AIMS Energy 8, 749-770. doi:10.3934/energy. 2020.5.749
Urrea, V., Ochoa, A., and Mesa, O. (2019). Seasonality of Rainfall in Colombia. Water Resources Research 55, 4149-4162. doi:10.1029/ 2018WR023316

Viviescas, C., Lima, L., Diuana, F. A., Vasquez, E., Ludovique, C., Silva, G. N., et al. (2019). Contribution of Variable Renewable Energy to increase energy security in Latin America: Complementarity and climate change impacts on wind and solar resources. Renewable and Sustainable Energy Reviews 113, 109232. doi:10. 1016/j.rser.2019.06.039

Widen, J. (2011). Correlations Between LargeScale Solar and Wind Power in a Future Scenario for Sweden. IEEE Transactions on Sustainable Energy 2, 177-184. doi:10.1109/TSTE.2010. 2101620

Xu, L., Wang, Z., and Liu, Y. (2017). The spatial and temporal variation features of wind-sun complementarity in China. Energy Conversion and Management 154, 138-148. doi:10.1016/j. enconman.2017.10.031 